\title{
Aneurisma de artéria pancreatoduodenal inferior: tratamento endovascular
}

\author{
Inferior pancreaticoduodenal artery aneurysm: endovascular approach \\ Patrick Bastos Metzger (1D, Kamilla Rosales Costa' (D), Simone Lessa e Silva², Alan Costa Gouveia ${ }^{3}$, \\ Murilo Quadro Berbert ${ }^{4}$, Milton Oliveira de Albuquerque Mello ${ }^{4}$, Fabrício Mascarenhas de Oliveira ${ }^{4}$, \\ Ricardo Fernandes Ferraz Melo ${ }^{4}$
}

\begin{abstract}
Resumo
Os aneurismas das artérias pancreatoduodenais constituem uma afecção rara. Seu diagnóstico é realizado, na maioria das vezes, em situações de emergência por complicações como a rotura, a qual está associada a altas taxas de mortalidade (21-26\%). A embolização do saco aneurismático é o tratamento de escolha, devido à sua alta efetividade e menor mortalidade. Neste artigo, é apresentado e discutido um caso de aneurisma de artéria pancreatoduodenal inferior com diagnóstico obtido durante investigação de sintomas gastrointestinais. O tratamento instituído foi a embolização com micromolas, com completa exclusão do aneurisma e boa evolução clínica.
\end{abstract}

Palavras-chave: aneurisma; procedimentos endovasculares; embolização terapêutica.

\begin{abstract}
Aneurysms of the pancreaticoduodenal arteries are a rare condition. In the majority of cases, diagnosis is made in emergency situations due to complications such as rupture, which is associated with high mortality rates (21-26\%). Embolization of the aneurysm sac is the treatment of choice, because of its high efficacy and lower mortality. This article presents and discusses a case of inferior pancreaticoduodenal artery aneurysm that was diagnosed during investigation of gastrointestinal symptoms. The treatment provided was microcoil embolization, with complete exclusion of the aneurysm and a good clinical course.
\end{abstract}

Keywords: aneurysm; endovascular procedures; therapeutic embolization.

Como citar: Metzger PB, Costa KR, Silva SL, et al. Aneurisma de artéria pancreatoduodenal inferior: tratamento endovascular. J Vasc Bras. 2021;20:e20200101. https://doi.org/10.1590/1677-5449.200101

1 Escola Bahiana de Medicina e Saúde Pública - EBMSP, Salvador, BA, Brasil.

${ }^{2}$ Obra Sociais Irmã Dulce - OSID, Hospital Santo Antônio, Salvador, BA, Brasil.

${ }^{3}$ Hospital Português, Salvador, BA, Brasil.

${ }^{4}$ Hospital da Bahia - HBA, Salvador, BA, Brasil.

Fonte de financiamento: Nenhuma.

Conflito de interesse: Os autores declararam não haver conflitos de interesse que precisam ser informados.

Aprovado pelo Comitê de Ética local.

Submetido em: Junho 10, 2020. Aceito em: Agosto 24, 2020

O estudo foi realizado no Hospital da Bahia (HBA), Salvador, BA, Brasil. 


\section{INTRODUÇÃO}

Os aneurismas de artérias viscerais (AAV) são raros, com uma incidência de $0,02 \%$ na população geral ${ }^{1-3}$. Entre eles, os aneurismas da artéria pancreatoduodenal (AAPD) são os menos frequentes, correspondendo a apenas $2 \%$ de todos os $\mathrm{AAV}^{4}$, sendo os aneurismas da artéria pancreatoduodenal inferior (AAPDi) os menos relatados ${ }^{1-3}$.

$\mathrm{O}$ uso da angiotomografia tem aumentado o diagnóstico de AAPD, devido a descobertas incidentais ${ }^{1-3}$. Por outro lado, atualmente a maioria dos AAPD ainda são descobertos e tratados em situações de emergência, com maior risco de complicações como hemoperitôneo e choque hipovolêmico, tendo taxas de ruptura aneurismática entre $45-53 \%$, na literatura ${ }^{3-5}$. Esses aneurismas são supridos por ramos colaterais com fluxo hiperdinâmico e estão frequentemente associados a estenoses oclusivas do tronco celíaco (TC) e da artéria mesentérica superior (AMS) ${ }^{3,4}$.

$\mathrm{O}$ tratamento endovascular é o método de tratamento preferencial, exceto em caso de suspeita de isquemia visceral, pacientes com a anatomia vascular hostil, ou quando houve falha de tratamento endovascular prévio. Nesses casos, é indicada a abordagem aberta ${ }^{2,3}$.

Relatamos um tratamento endovascular de um volumoso AAPDi verdadeiro, sem estenoses significativas (estenose $>60 \%$ ) nos troncos arteriais principais, utilizando navegação superseletiva com microcateteres e embolização com micromolas.

\section{DESCRIÇÃO DO CASO}

Uma mulher de 62 anos, hipertensa, diabética tipo 2, obesa e dislipidêmica, estava em investigação ambulatorial por empachamento pós-prandrial com gastroenterologista. Negava história familiar ou pregressa de doenças reumatológicas, assim como episódios de hemorragia digestiva, dor pós-prandial, traumas abdominais, ingestão de bebidas alcoólicas, história prévia de pancreatite, ou perda de peso no período. A angiotomografia demonstrou volumoso AAPDi, com $54 \mathrm{x}$ $60 \mathrm{~mm}$, conectando a AMS com a artéria gastroduodenal. AAMS não apresentava placa ostial, e o TC apresentava placa ostial determinando estenose aproximada de $20 \%$ (Figura 1). Foi realizado ecocardiograma transtorácico sem evidências de alterações patológicas.

Uma arteriografia seguida de navegação superseletiva com microcateteres e embolização com micromolas foi a opção terapêutica adotada. O procedimento foi realizado em sala de hemodinâmica, sob anestesia local e sedação consciente, com acesso femoral e uso de introdutor $5 \mathrm{Fr}$ (Terumo Medical, Somerset, EUA). Após estudo arteriográfico com cateterização sequencial e seletiva do TC e da AMS, optou-se por cateterização da AMS e navegação superseletiva com microcateter via artéria pancreatoduodenal inferior, do volumoso aneurisma. Foi realizada embolização com uso do Microcateter Rebar-18® (ev3; Plymouth, EUA) e do microfio-guia SilverSpeed-16 ${ }^{\circledR}$ (ev3; Plymouth, Minnesota, EUA), além de embolização com 6 micromolas Axium ${ }^{\circledR}$ Prime 3D (ev3; Plymouth, EUA) destacáveis de $18 \mathrm{~mm}$ x $40 \mathrm{~cm}$. Após completa embolização do aneurisma, foi realizado novo estudo arteriográfico seletivo via TC e AMS, que revelou completa exclusão do AAPDi, assim como melhora da perfusão das alças intestinais (Figura 2).

O pós-operatório foi realizado em unidade de terapia intensiva, com boa evolução, e houve alta hospitalar no segundo dia após o procedimento. $\mathrm{O}$ controle por angiotomografia após 1 mês mostrou perviedade da AMS e TC, com exclusão completa do AAPDi. Durante o acompanhamento ambulatorial de 1 ano, a paciente apresentou melhora das queixas pós-prandiais previamente referidas (Figura 3).
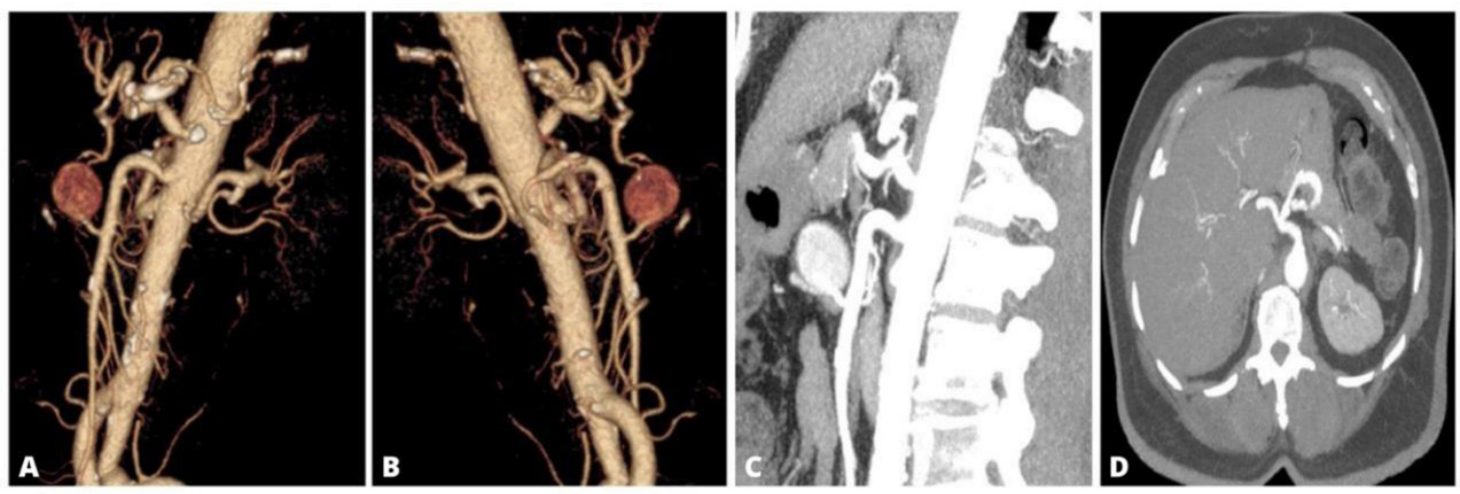

Figura 1. (A) Reconstrução angiotomográfica volumétrica em oblíqua anterior esquerda demonstrando volumoso aneurisma de artéria pancreatoduodenal inferior; (B) Reconstrução volumétrica angiotomográfica em oblíqua anterior direita demonstrando comunicações com tronco celíaco (TC), via artéria gastroduodenal, e com artéria mesentérica superior, via artéria pancreatoduodenal inferior; (C) Corte angiotomográfico axial demonstrando placa ostial de TC; (D) Corte angiotomográfico sagital demonstrando placa ostial em TC determinando estenose aproximada de $20 \%$. 

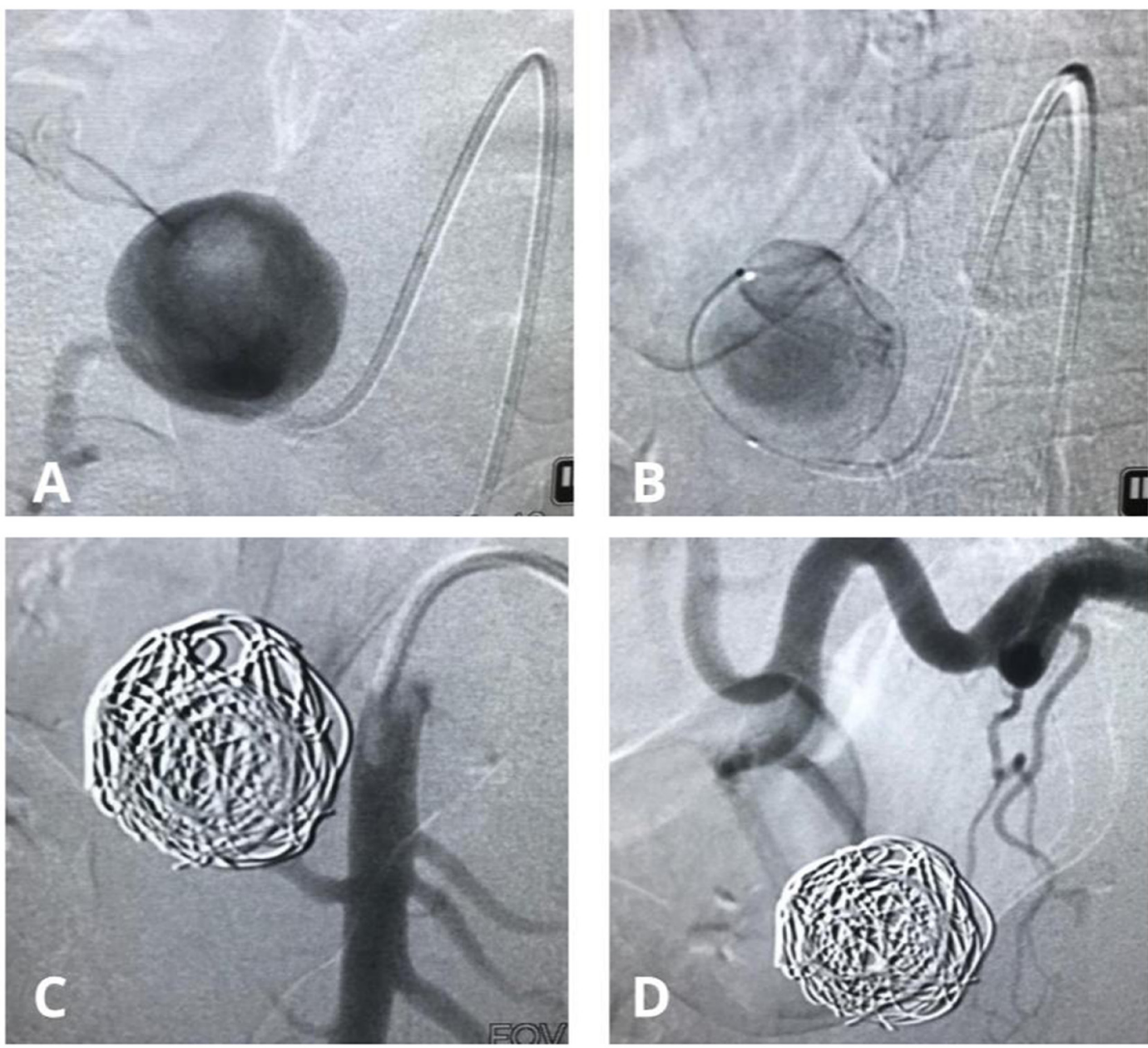

Figura 2. (A) Arteriografia via artéria mesentérica superior (AMS), com cateterização seletiva; (B) Seletivação com microcateter do volumoso aneurisma da artéria pancreatoduodenal inferior; (C) Arteriografia seletiva da AMS pós-embolização com micromolas, demonstrando exclusão completa do saco aneurismático; (D) Arteriografia seletiva do tronco celíaco, pós-embolização com micromolas, demonstrando exclusão completa do saco aneurismático.

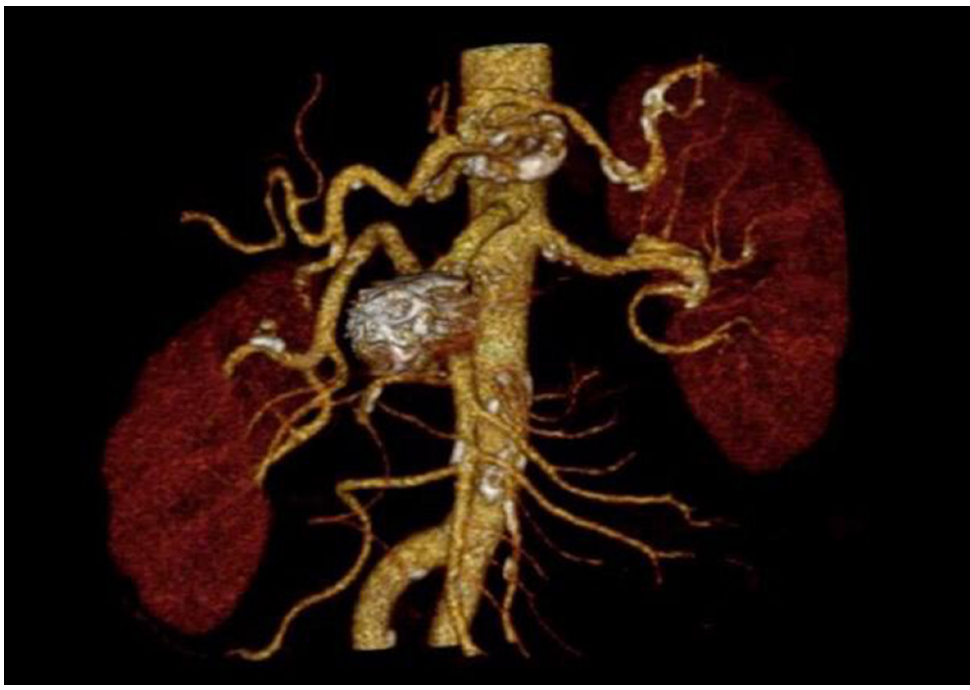

Figura 3. Reconstrução angiotomográfica volumétrica pós-operatória demonstrando ausência de enchimento do aneurisma, com sua exclusão completa. 


\section{DISCUSSÃO}

O AAPDi é extremamente raro, mas provavelmente existe subnotificação, uma vez que seus sintomas são inespecíficos e sua localização dificulta o diagnóstico ${ }^{5,6}$. Acomete ambos os sexos em igual incidência ${ }^{5,6}$, e a média de idade de maior frequência diagnóstica é aos $60 \operatorname{anos}^{6}$. A principal causa dos AAPDi verdadeiros é a oclusão ou a estenose de um dos troncos arteriais principais, seja AMS ou TC, sendo este último o mais comumente acometido (80\%); além disso, o aneurisma está relacionado com a doença arterial oclusiva dos óstios desses troncos em $50-80 \%$ dos $\operatorname{casos}^{3,7,8}$. Após esse evento, a velocidade do fluxo sanguíneo se eleva para as arcadas arteriais pancreáticas, justificando a dilatação progressiva do lúmen arterial (hipótese de circulação colateral hiperdinâmica $)^{2,5,8,9}$. Outras causas relacionadas são aterosclerose, colagenoses e infecções ${ }^{5,10}$. Estas últimas foram consideradas por muitos anos como a principal causa de AAPDi $(60 \%)$; no entanto, estudos recentes revelam que a doença aterosclerótica é a causa mais comum, sendo a etiologia infecciosa responsável por $4,8 \%$ dos casos diagnosticados, e não mais da metade, como se acreditava anteriormente ${ }^{10}$. A paciente em questão foi diagnosticada com AAPDi verdadeiro, pois não apresentou critérios para suspeição de infecção micótica ou de colagenoses, e também não apresentava estenose ostial de TC ou AMS, afastando a hipótese de circulação colateral hiperdinâmica ${ }^{2,5,8,9}$. Diante disso, a provável causa do aneurisma dessa paciente é a doença aterosclerótica.

A principal complicação associada ao AAPDi é a ruptura, cuja incidência e mortalidade variam de 45 a $62 \%$ e de $21-26 \%$, respectivamente ${ }^{3,11,12}$. Uma parcela significativa dos diagnósticos dessa afecção é realizada na emergência, com sintomas de epigastralgia ou dor abdominal que irradia para o dorso, os quais podem estar associados a sinais de choque ou hemoperitônio secundários à rotura aneurismática ${ }^{10}$. Essa complicação independe da dimensão do aneurisma ${ }^{9}$.

A paciente relatada apresentou queixa gastrointestinal de empachamento em investigação ambulatorial, com posterior diagnóstico de AAPDi após angiotomografia de abdome. Dor abdominal inespecífica, náuseas, vômitos, icterícia e sangramento gastrointestinal são sintomas que podem estar associados nesses casos. Aneurismas de origem infecciosa podem ocasionar febre. Ao exame físico, uma massa pulsátil em abdome ou sopro abdominal podem ser encontrados, porém são raros. A frequente presença dessas manifestações em pacientes com AAPDi os distingue daqueles com outros tipos de aneurismas de artérias esplâncnicas, em sua maioria assintomáticos $(90 \%)^{10}$; entretanto, o diagnóstico na maioria dos casos é realizado só após a rotura do aneurisma. Por isso, diante da sua alta mortalidade, o AAV deve ser um diagnóstico diferencial para todo paciente com dor abdominal súbita associada a anemia ${ }^{9}$. Os exames complementares que auxiliam no diagnóstico são: ultrassonografia-Doppler abdominal, que constitui um método disponível e de baixo custo; angiografia por ressonância magnética; e angiotomografia (exame padrão-ouro), que fornece informações necessárias tanto para a o diagnóstico como para a decisão terapêutica ${ }^{9,10}$.

Por se tratar de afecção rara, seu tratamento tem indicações controversas na literatura, sendo necessária a avaliação de fatores como idade, apresentação clínica, características do aneurisma, comorbidades associadas e fatores de risco ${ }^{3,5,10}$. Alguns consensos entre os autores quanto à indicação de correção de aneurismas verdadeiros são: diâmetro superior a 20-25 $\mathrm{mm}$, mesmo assintomático; pacientes sintomáticos; pacientes do sexo feminino em idade fértil; e aneurismas associados a processo inflamatório ou infeccioso, doença do tecido conjuntivo, vasculite ou doenças congênitas, devido ao alto risco de rotura ${ }^{5,6,9,13,14}$. Já o pseudoaneurisma deve ser abordado de imediato após o seu diagnóstico, devido ao elevado risco de rotura $^{5,7,9}$. Como opções terapêuticas disponíveis, têm-se o tratamento cirúrgico convencional (reparo aberto) e a abordagem endovascular. A cirurgia convencional é indicada para pacientes hemodinamicamente instáveis, quando há necessidade de revascularização do fluxo sanguíneo, e em casos de aneurisma em territórios inacessíveis pela técnica endovascular ou de falha após embolização. Essa técnica consiste na ligadura proximal e distal da artéria, exérese do aneurisma e reconstrução da artéria. O reparo aberto, além de ser um procedimento mais invasivo, apresenta limitações, devido à dificuldade de acesso ao aneurisma, além de estar associado a taxas de mortalidade entre $15-23,7 \%$, 5,10 .

Com a evolução das técnicas e dos dispositivos disponíveis, o tratamento endovascular com a embolização do aneurisma tornou-se o tratamento de escolha nesses casos, devido à sua alta efetividade (67$100 \%)$, menor taxa de mortalidade $(2,7 \%)$, menor risco de rotura, menor dor pós-operatória, menor tempo de recuperação após o procedimento, com consequente retorno mais precoce às atividades cotidianas, além de constituir uma opção para pacientes com histórico de cirurgias abdominais que tenham um abdome hostil ${ }^{5,9,10}$. No entanto, alguns determinantes limitam o seu uso: baixa disponibilidade em centros de emergência, risco de lesões secundárias ao acesso e embolização de órgãos-alvo, assim como elevado custo ${ }^{9}$.

As opções de embolização envolvem a utilização de cianocrilato, molas e stents revestidos ou convencionais, podendo, em algumas ocasiões, serem associados ${ }^{7,9,10}$. Outras opções de tratamento percutâneo são o desvio de fluxo e a injeção percutânea de trombina ${ }^{9,15,16}$. A 
escolha da técnica baseia-se na necessidade de preservar a artéria nutridora, nas características anatômicas e no tipo do aneurisma, além da tortuosidade da artéria nativa. $\mathrm{O}$ tratamento adequado do aneurisma é garantido pela embolização completa de todas as artérias de entrada e saída ${ }^{9,15,16}$.

A paciente deste relato apresentou um AAPDi oligossintomático com dimensões de $54 \times 60 \mathrm{~mm}$, sem rotura. Diante da sua compatibilidade com os critérios de indicação para a abordagem terapêtica mais difundidos na literatura, e também pelo elevado risco de rotura, foi indicado o tratamento eletivo por meio da técnica

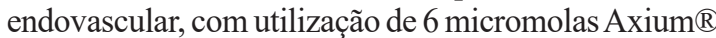
Prime 3D destacáveis (ev3; Plymouth, Minnesota, USA), com trombose permanente no saco aneurismático e exclusão deste. A embolização foi completa, confirmada no intraoperatório pela angiografia seletiva do TC e da AMS, com melhora do fluxo sanguíneo intestinal e boa evolução pós-operatória, inclusive com melhora dos sintomas clínicos prévios. Angiotomografia de controle com 1 mês foi realizada confirmando a trombose total do aneurisma e sem evidências de enchimento deste.

\section{REFERÊNCIAS}

1. Vandy FC, Sell KA, Eliason JL, Coleman DM, Rectenwald JE, StanleyJC Pancreaticoduodenal and gastroduodenal artery aneurysms associated with celiac artery occlusive disease. Ann Vasc Surg. 2017;41:32-40. http://dx.doi.org/10.1016/j.avsg.2016.09.018. PMid:28238920.

2. Jacobs CR, Crawford JD, Fatima J. Hybrid management approach for superior mesenteric artery and branch aneurysms. J Vasc Surg Cases Innov Tech. 2019;5(4):521-4. http://dx.doi.org/10.1016/j. jvscit.2019.10.002. PMid:31909308.

3. Bonardelli $S$, Spampinato B, Ravanelli $M$, et al. The role of emergency presentation and revascularization in aneurysms of the peripancreatic arteries secondary to celiac trunk or superior mesenteric artery occlusion. J Vasc Surg. 2020;72(1):46S-55S http://dx.doi.org/10.1016/j.jvs.2019.11.051. PMid:32093911.

4. Kitaoka T, Deguchi J, Kamiya C, Suzuki J, Sato O. Pancreaticoduodenal artery aneurysm formation with superior mesenteric artery stenosis. Ann Vasc Dis. 2014;7(3):312-5. http://dx.doi.org/10.3400/avd. cr.14-00040. PMid:25298835.

5. Fetti AC, Puia VR, Comsa M, Stanca MH, Puia A, Al Hajjar N. Inferior pancreatico-duodenal artery aneurysm with superior mesenteric artery stenosis. J Gastrointestin Liver Dis. 2020;29(1):115-8. http:// dx.doi.org/10.15403/jgld-390. PMid:32176753.

6. Nasser F, Affonso BB, Jesus-Silva SG, et al. Endovascular treatment for superior mesenteric artery pseudoaneurysm: case report. J Vasc Bras. 2010;9(3):182-5. http://dx.doi.org/10.1590/ S1677-54492010000300016.

7. Sutton D, Lawton G. Coeliac stenosis or occlusion with aneurysm of the collateralsupply. Clin Radiol. 1973;24(1):49-53. http://dx.doi. org/10.1016/S0009-9260(73)80114-X. PMid:4723494.

8. Kadir S, Athanasoulis CA, Yune HY, Wilkov H. Aneurysms of the pancreaticoduodenal arteries in association with celiac axis occlusion. Cardiovasc Radiol. 1978;1(3):173-7. http://dx.doi. org/10.1007/BF02552029. PMid:743713.

9. Kallamadi R, Demoya MA, Kalva SP. Inferior pancreaticoduodenal artery aneurysms in association with celiac stenosis/occlusion. Semin Intervent Radiol. 2009;26(3):215-23. http://dx.doi. org/10.1055/s-0029-1225671. PMid:21326566.
10. Obara $H$, Kentaro $M$, Inoue $M$, Kitagawa Y. Current management strategies for visceral artery aneurysms: an overview. Surg Today. 2020;50(1):38-49. http://dx.doi.org/10.1007/s00595-019-01898-3. PMid:31620866.

11. Bortoluzzi C, Franzon O, Viana R, Mattiello L, Stahelin AHS. Duplo aneurisma de artéria mesentérica superior: relato de caso. J Vasc Bras. 2018;17(4):328-32. http://dx.doi.org/10.1590/1677-5449.005818. PMid:30787952.

12. Moore E, Matthews MR, Minion DJ, et al. Surgical management of peripancreatic arterial aneurysms. J Vasc Surg. 2004;40(2):247-53. http://dx.doi.org/10.1016/j.jvs.2004.03.045. PMid:15297817.

13. de Perrot M, Berney T, Deleaval J, Buhler L, Mentha G, Morel P. Management of true aneurysms of the pancreaticoduodenal arteries. Ann Surg. 1999;229(3):416-20. http://dx.doi.org/10.1097/00000658199903000-00016. PMid:10077055.

14. Habib N, Hassan S, Abdou R, et al. Gastroduodenal artery aneurysm, diagnosis, clinical presentation and management: a concise review. Ann Surg Innov Res. 2013;7(1):4. http://dx.doi. org/10.1186/1750-1164-7-4. PMid:23587203.

15. Tulsyan N, Kashyap VS, Greenberg RK, et al. The endovascular management of visceral artery aneurysms and pseudoaneurysms. J Vasc Surg. 2007;45(2):276-83. http://dx.doi.org/10.1016/j. jvs.2006.10.049. PMid:17264002.

16. Hemp JH, Sabri SS. Endovascular management of visceral arterial aneurysms. Tech Vasc Interv Radiol. 2015;18(1):14-23. http:// dx.doi.org/10.1053/j.tvir.2014.12.003. PMid:25814199.

Correspondência
Patrick Bastos Metzger
Escola Bahiana de Medicina e Saúde Pública - EBMSP
Alameda de Pádua, 25 - Pituba
CEP 41830-480 - Salvador (BA), Brasil
Tel.: (71) 99977-6356
E-mail: patrickvascular@gmail.com

Informações sobre os autores

PBM - Doutor em Medicina, Universidade de São Paulo; Cirurgião Vascular e Endovascular, Sociedade Brasileira de Angiologia e Cirurgia Vascular e Radiologista Intervencionista, Colégio Brasileiro de Radiologia.

KRC - Acadêmica, $9^{\circ}$ semestre de Medicina, Escola Bahiana de Medicina e Saúde Pública (EBMSP). SLS - Preceptora, residência de Geriatria, Hospital Santo Antônio, Obra Sociais Irmã Dulce. ACG - Cirurgião Vascular, Sociedade Brasileira de Angiologia e Cirurgia Vascular. MQB e MOAM - Cirurgiões Vasculares e Endovasculares, Sociedade Brasileira de Angiologia e Cirurgia Vascular.

FMO - Cirurgião Vascular, Sociedade Brasileira de Angiologia e Cirurgia Vascular Radiologista Intervencionista, Colégio Brasileiro de Radiologia.

RFFM - Cirurgião Geral, Ministério da Educação e Cultura.

Contribuições dos autores Concepção e desenho do estudo: PBM Análise e interpretação dos dados: KRC, PBM, SLS Coleta de dados: PBM, ACG, MQB, MOAM, RFFM, FMO Redação do artigo: KRC e PBM Revisão crítica do texto: PBM

Aprovação final do artigo*: KRC, PBM, SLS, ACG, MQB, MOAM, RFFM, FMO

Análise estatística: KRC, PBM, SLS Responsabilidade geral pelo estudo: PBM

*Todos os autores leram e aprovaram a versão final submetida ao J Vasc Bras. 